\title{
Beneficial and harmful consequences of prepartum and postpartum antidepressant exposure
}

\author{
Corrado Barbui, Giovanni Ostuzzi
}

Department of Public Health and Community Medicine, Section of Psychiatry, WHO Collaborating Centre for Research and Training in Mental Health and Service Evaluation, University of Verona, Verona, Italy

Correspondence to Corrado Barbui, corrado.barbui@univr.it

\section{SETTING THE SCENE}

Since women are twice as likely as men to experience depression, and depression mainly occurs during their reproductive years, high rates of depression during pregnancy and postpartum are expected. ${ }^{1}$ Epidemiological data suggest that the prevalence of major depression in pregnant women is in the range of $3-5 \%$, and the combined prevalence of major and minor depression can reach $8-11 \%{ }^{2}{ }^{3}$ Postpartum depression, which indicates a major depressive episode that occurs within the first 4 weeks after delivery or, using broader clinical criteria, within the first year after childbirth, may affect up to $13 \%$ of women. ${ }^{45}$

As a consequence, a relevant and growing number of women are exposed to antidepressants (ADs) during pregnancy, after delivery and over the following months. On average, $1-8 \%$ of women receive $A D$ medication during pregnancy, with higher prevalence of use during the first trimester. ${ }^{1}$ In the USA, a recent study showed that prenatal visits associated with $A D$ prescriptions had increased from $0.7 \%$ (20022006) to $2.1 \%$ (2007-2010). ${ }^{6}$ In Denmark, a 16-fold increase in exposure rates between 1997 and 2010 was observed, from a rate of $0.2 \%$ in 1997 to $3.2 \%$ in $2010 .^{7}$ After delivery and during the first year after childbirth the prevalence of $A D$ prescriptions may steadily increase, as recently suggested by a Danish population-based prevalence study. ${ }^{8}$

The combination of depression and $A D$ use during pregnancy and postpartum has been associated with harmful consequences in terms of neonatal and child development outcomes.

\section{THE PROBLEM}

An increased risk of physical and emotional/behavioural problems has consistently been observed in the offspring of mothers suffering from depression in the perinatal period. ${ }^{9} 10$ Probably, complex interactions between several pathways may explain this phenomenon, including biological mechanisms (eg, fetal exposure to maternal stress hormones), behavioural and emotional features (eg, poorer prenatal care and attachment style of depressed mothers) and genetic predisposition transmitted to the offspring. ${ }^{10}$ In addition to depression during pregnancy and postpartum, exposure to ADs is another major concern.

From a clinical point of view, treatment of depression with ADs during pregnancy and postpartum raises two challenging questions: in such a special population is efficacy of ADs similar as compared with the general population? Is the potentially beneficial effect of ADs for the mother outweighed by harmful consequences for the newborn as ADs cross the placenta and are present in amniotic fluid and mother's milk?

\section{THE SELECTED STUDIES}

In this issue of $E B M H$, three studies are reviewed that expand current knowledge on the efficacy of ADs in women with postpartum depression, ${ }^{11}$ risk of pulmonary hypertension ${ }^{12}$ and risk of autism spectrum disorders $^{13}$ in the offspring of women exposed to $A D$ in pregnancy. Of note, one study employed a cohort design, while the other two are systematic reviews of randomised or observational studies (table 1).

\section{BENEFICIAL EFFECTS OF ANTIDEPRESSANTS DURING PREGNANCY AND POSTPARTUM}

Whether $A D$ s are effective in treating depression in pregnant women cannot be directly assessed by means of randomised trials, as these would be deemed as unethical. It can only be indirectly inferred from randomised trials performed in general populations of individuals with major depression. These studies always exclude pregnant women and usually lump together data on men and women. However, assuming that pregnant women have a similar response to $A D$ treatment as compared with the general population, randomised evidence suggests that the average benefit of these drugs is of small magnitude ${ }^{14}$ although it is possible that current approaches to estimation of the benefits of $A D$ treatments may underestimate their clinical significance. ${ }^{15}$ Roughly, 53 of 100 depressed patients treated with an $A D$ respond to treatment, as compared with 42 of 100 depressed patients treated with placebo, which yields an absolute difference of 11 patients out of $100 .{ }^{16}$ We additionally know that $A D$-placebo differences increase with increasing baseline severity and the difference becomes large enough to be clinically important only in moderate-to-severe major depression. Based on this indirect evidence, therefore, it is generally assumed that $A D$ drugs are effective in treating moderate-to-severe depression in pregnant women.

It is true that there is no randomised data in pregnant women; however, in the postnatal period more robust evidence is available and it has recently been systematically reviewed by De Crescenzo et al. ${ }^{11}$ Out of six included studies, only three compared one selective serotonin reuptake inhibitor (SSRI) with placebo. Of these three studies, information on responders was available in two studies only: overall, 21 of 35 depressed women treated with an SSRI responded to treatment, as compared with 15 of 35 depressed women treated with placebo. This yielded an absolute difference of $11 \%$ (95\% Cl $-4 \%$ to $26 \%$ ). In addition, when compared with psychological treatments, pharmacotherapy with SSRIs failed to show any significant difference.

Table 1 Main characteristics of three recent studies which investigated the beneficial and harmful consequences of prepartum and postpartum antidepressant exposure

\begin{tabular}{|c|c|c|c|c|}
\hline \multirow[b]{2}{*}{ Study } & \multirow{2}{*}{$\begin{array}{l}\text { Exposure } \\
\text { Antidepressants }\end{array}$} & \multicolumn{2}{|l|}{ Outcome } & \multirow[b]{2}{*}{ Study design } \\
\hline & & Children & Mothers & \\
\hline Hviid et $a l^{13}$ & Pregnancy & Autism & & Observational study (626 875children) \\
\hline Grigoriadis et al ${ }^{12}$ & Pregnancy & $\begin{array}{l}\text { Pulmonary } \\
\text { hypertension }\end{array}$ & & $\begin{array}{l}\text { Systematic review of seven observational studies (for characteristics of included studies, see } \\
\text { http://www.bmj.com/content/suppl/2014/01/14/bmj.f6932.DC2/gris014934.ww1_default.pdf) }\end{array}$ \\
\hline De Crescenzo et al ${ }^{11}$ & Postpartum & & $\begin{array}{l}\text { Depressive } \\
\text { symptoms }\end{array}$ & Systematic review of six randomised studies (595 patients) \\
\hline
\end{tabular}


It is striking to note that only few randomised trials have been conducted in this patient population, with very small sample size. This would lead to the conclusion that direct evidence on the efficacy of ADs in postpartum depression is virtually absent. However, putting these findings into the wider context of the whole evidence base on the efficacy of $A D s$ in major depression, it seems interesting to note that the magnitude of $A D$ effect, in terms of absolute risk difference (ARD), is similar between women with postpartum depression and the general population, and that lack of statistical significance in studies conducted in postpartum depression is likely the consequence of a power problem. Clinically, therefore, current best evidence would indicate that women with postpartum depression have a similar response to $A D$ treatment as compared with the general population.

\section{HARMFUL EFFECTS OF ANTIDEPRESSANTS DURING PREGNANCY: NEONATAL OUTCOMES}

It has been noted that ADs are the most studied drugs during pregnancy, with more than 30000 neonatal outcomes following exposure during pregnancy documented in the peer-reviewed literature. ${ }^{17}$ The results of these studies are sometimes conflicting because of different interpretation of statistical versus clinical significance. However, in terms of major malformations, no specific pattern has been consistently associated with ADs, with the possible exception of paroxetine and cardiac malformations. ${ }^{18}$ It is generally reported that $A D$ exposure may be associated with a significant increased risk of spontaneous abortion, preterm birth and infants born weighing less than $2500 \mathrm{~g}^{17} 19$

In addition, there might be a possible increased risk of persistent pulmonary hypertension of the newborn, but this finding is rather controversial. ${ }^{17}$ Recently, in order to expand current knowledge on this harmful outcome, Grigoriadis et al ${ }^{12}$ carried out a systematic review and meta-analysis of observational studies that examined the risk for persistent pulmonary hypertension of the newborn associated with antenatal $A D$ exposure. Seven studies were included in the quantitative analysis. It was found that while exposure to SSRls in early pregnancy was not associated with persistent pulmonary hypertension of the newborn (data from three studies), exposure in late pregnancy was associated with more than a twofold increase (five studies). Effects were not significant for any of the moderator variables examined, including study design, congenital malformations and meconium aspiration.

As often is the case with systematic reviews and meta-analyses, the main limitations are those of the original studies, which have been clearly analysed by the study authors. An additional concern, when meta-analytical techniques are applied, is whether pooling is appropriate. Studies must be homogeneous in terms of populations, exposure variables, comparisons and outcomes. This is a challenging issue when observational studies are pooled, as their design may markedly differ in relation to the different sources of data that are used. If studies are not similar, then heterogeneity is usually detected by visually checking in the forest plot the degree of overlap of the Cls around the point estimates calculated for each included studies. Additionally, heterogeneity may be investigated statistically. $^{20} 21$ In this analysis, substantial heterogeneity was detected, despite a small number of included studies, which might suggest caution in pooling data. The reasons for this significant heterogeneity remains partially unknown, as the characteristics of the primary studies did not allow proper evaluation of a number of potential sources, including differences in the definition of early versus late exposure to SSRIs, maternal obesity, preterm birth and other factors.

Clinically, the statistically significant increased risk should be interpreted taking into account that persistent pulmonary hypertension is a relatively rare outcome, affecting on average 1.9/1000 newborns. Therefore, considering an increased relative risk of 2.5 , as reported by this meta-analysis, it is possible to estimate an absolute risk, associated with SSRI exposure, of $1.9 \times 2.5$, which is 4.75 , and an ARD of
$4.75-1.9$, which is $2.85 / 1000$ newborns (ie, $0.285 \%$ or 0.00285 ). The inverse of the ARD (ie, 1/ARD) provides the number of women that need to be exposed to SSRIs during pregnancy to have one additional newborn with pulmonary hypertension, which are 351 (ie, 1/0.00285). In other words, a doctor would need to treat with SSRls 351 pregnant women to have one additional newborn with pulmonary hypertension. These numbers prompted the authors to cautiously conclude that although the statistical association was significant, clinically the absolute risk of persistent pulmonary hypertension of the newborn remained low even in the context of late exposure to SSRIs. ${ }^{12}$

\section{HARMFUL EFFECTS OF ANTIDEPRESSANTS DURING PREGNANCY: CHILD DEVELOPMENT OUTCOMES}

While the majority of studies on ADs during pregnancy are focused on teratogenic effects, delivery complications and neonatal toxicity, current knowledge on the potential harmful effects on child development, including autism spectrum disorders (ASDs) and emotional or behavioural problems in infancy and adolescence, are very unsatisfactory. Recently, a research team from Denmark performed a large populationbased and register-based prospective cohort study to shed further light on this compelling issue. ${ }^{13}$ Researchers included only singleton births in a 10-year period, and excluded conditions associated with an increased risk of ASDs (eg, congenital rubella syndrome or some genetic disorders in parents). Information on a number of potential confounders was additionally collected (eg, maternal psychiatric diagnosis, age at the onset of pregnancy, parity, etc). Children were prospectively followed from birth until 5-10 years of age. Out of a total study cohort of 626875 live births, 6068 children (1.0\%) were exposed to SSRIs during pregnancy. In terms of risk of developing ASD, children of women exposed to SSRls before and during pregnancy were more likely to receive a diagnosis of ASD. However, this significant association disappeared after adjusting for the following variables: mother's age at birth, country of origin, place of residence, parity, psychiatric diagnoses before delivery, other drug use during pregnancy, smoking status during pregnancy, employment status and level of education. Similar results were found with children of women exposed to SSRI only during pregnancy.

In the present observational study, considering that the reference category was constituted by women with no SSRI exposure, confounding by indication might have occurred, as maternal depression is a risk factor for developing ASD. This might explain the finding that significant associations disappeared after adjusting for psychiatric diagnosis and, intriguingly, that in comparison with no SSRI exposure, children of women exposed to SSRls only before pregnancy (which could be considered as a proxy of suffering from depression during pregnancy) were at increased risk of developing ASD. Despite maternal depression being included in the analysis as a confounding variable, information on maternal diagnoses was available only from psychiatric hospitals and psychiatric units, but not from primary care settings, where the majority of cases of maternal depression is expected to be recognised. It is therefore possible that residual confounding by indication might have occurred. A second concern refers to the study cohort. Although it is representative of the Denmark population, it may not be representative of other populations, as shown by the prevalence of pregnancy-related use of SSRls, which was very low in the present study, as compared, for example, with the USA. ${ }^{22}$ The same applies to the prevalence of autism, lower in this study as compared with, for example, the USA. ${ }^{13}$

A third reason for concern is the exposure variable, as the present study employed SSRI prescription as a proxy of SSRI use. The general issue that a relevant proportion of medicines prescribed for people with chronic conditions are not taken might be particularly challenging in this study, as depressed women might decide not to take a prescribed treatment with SSRls, when pregnant, more often than depressed women who are not pregnant. A final concern is that length of 
follow-up was 5-10 years, which means that some children were followed for 10 years after birth while for others follow-up was truncated at 5 years. Considering the relatively high rates of ASD diagnoses in scholar age ${ }^{23}$ it is possible that some children were erroneously categorised as non-cases because a diagnosis of ASD was made after 5 years of age. Clearly, it is difficult to speculate on whether this under recognition might have somewhat hampered the analyses.

It is worth noting that, according to the study authors, these results are not conclusive, as an increased risk of ASDs cannot be completely ruled out: on the basis of the upper boundary of the $\mathrm{Cl}$ of the main analysis (which is 1.61), it cannot be excluded that SSRI exposure increases the relative risk of developing ASDs up to $61 \%$.

\section{CONCLUDING REMARKS}

The three studies briefly described in the present commentary outline the value of weighting the potential beneficial effects associated with $A D$ use in pregnancy and postpartum against a number of potentially harmful consequences. This cautious approach should be individualised on a case-by-case basis, employing shared decision-making techniques that involve the mother and experts in psychiatry, obstetrics and gynaecology. It should be recognised that the treatment of women with ADs during pregnancy and postpartum is challenging and complex. ${ }^{15}$

Doctors should be aware that recent data refer almost exclusively to the SSRls, the most commonly prescribed ADs in Western countries. However, in several low-income and middle-income countries tricyclic and related ADs are still widely prescribed. In addition, in depressed patients with no satisfactory response to the SSRIs, switching to a different $A D$ is a frequent therapeutic option. Thus, $A D$ drugs not belonging to the SSRI class are still widely prescribed globally, and therefore a clearer understanding of whether some of the risks associated with SSRI exposure similarly apply to other $\mathrm{AD}$ classes is a public health priority.

In general, treatment guidelines suggest that in women with mild-to-moderate depression without a history of recurrent or severe depression, or women with depression related to specific adjustments or stressors, psychotherapy with a trained provider is a first-line intervention. ${ }^{1}$ In women with more severe depression a thorough risk-benefit discussion of ADs in general and the specific medication in particular is warranted, taking into consideration that paroxetine is generally contraindicated. In postpartum depression pharmacological treatment recommendations for women who are lactating must include discussion with the patient regarding the benefits of breastfeeding, risks of $A D$ use during lactation and risks of untreated illness. Doctors should consider that treatment decisions must be guided by the woman's preference and should be contextualised to local healthcare, social and economic circumstances.

Competing interests None.

doi:10.1136/eb-2014-101884

\section{REFERENCES}

1. Chaudron LH. Complex challenges in treating depression during pregnancy. Am J Psychiatry 2013;170:12-20.

2. Andersson L, Sundstrom-Poromaa I, Wulff M, et al. Depression and anxiety during pregnancy and six months postpartum: a follow-up study. Acta Obstet Gynecol Scand 2006;85:937-44.

3. Andersson L, Sundstrom-Poromaa I, Bixo M, et al. Point prevalence of psychiatric disorders during the second trimester of pregnancy: a population-based study. $A m \mathrm{~J}$ Obstet Gynecol 2003;189:148-54.

4. Dennis CL, McQueen K. The relationship between infant-feeding outcomes and postpartum depression: a qualitative systematic review. Pediatrics 2009;123: e736-51.

5. Guille C, Newman R, Fryml LD, et al. Management of postpartum depression. J Midwifery Womens Health 2013;58:643-53.

6. Meunier MR, Bennett IM, Coco AS. Use of antidepressant medication in the United States during pregnancy, 2002-2010. Psychiatr Serv 2013;64:1157-60.

7. Jimenez-Solem E, Andersen JT, Petersen M, et al. Prevalence of antidepressant use during pregnancy in Denmark, a nation-wide cohort study. PLOS ONE 2013;8: e63034.

8. Munk-OIsen T, Gasse C, Laursen TM. Prevalence of antidepressant use and contacts with psychiatrists and psychologists in pregnant and postpartum women. Acta Psychiatr Scand 2012;125:318-24.

9. Raposa E, Hammen C, Brennan P, et al. The long-term effects of maternal depression: early childhood physical health as a pathway to offspring depression. J Adolesc Health 2014;54:88-93.

10. Leis JA, Heron J, Stuart EA, et al. Associations between maternal mental health and child emotional and behavioral problems: does prenatal mental health matter? J Abnorm Child Psychol 2014;42:161-71.

11. De Crescenzo F, Perelli F, Armando M, et al. Selective serotonin reuptake inhibitors (SSRIs) for post-partum depression (PPD): a systematic review of randomized clinical trials. J Affect Disord 2014;152:39-44.

12. Grigoriadis S, Vonderporten EH, Mamisashvili L, et al. Prenatal exposure to antidepressants and persistent pulmonary hypertension of the newborn: systematic review and meta-analysis. BMJ 2014;348:f6932.

13. Hviid A, Melbye M, Pasternak B. Use of selective serotonin reuptake inhibitors during pregnancy and risk of autism. N Engl J Med 2013;369:2406-15.

14. Ioannidis JP. Effectiveness of antidepressants: an evidence myth constructed from a thousand randomized trials? Philos Ethics Humanit Med 2008;3:14.

15. Adli M, Hegerl U. Do we underestimate the benefits of antidepressants? Lancet 2014;383:1361-2.

16. Barbui C, Furukawa TA, Cipriani A. Effectiveness of paroxetine in the treatment of acute major depression in adults: a systematic re-examination of published and unpublished data from randomized trials. CMAJ 2008;178:296-305.

17. Einarson A. Antidepressant use during pregnancy: navigating the sea of information. Can Fam Physician 2013;59:941-3.

18. Reis $\mathbf{M}$, Kallen B. Delivery outcome after maternal use of antidepressant drugs in pregnancy: an update using Swedish data. Psychol Med 2010;40:1723-33.

19. Yonkers KA, Blackwell KA, Glover J, et al. Antidepressant use in pregnant and postpartum women. Annu Rev Clin Psychol 2014;10:369-92.

20. Purgato M, Adams CE. Heterogeneity: the issue of apples, oranges and fruit pie. Epidemiol Psychiatr Sci 2012;21:27-9.

21. Ioannidis JP, Patsopoulos NA, Evangelou E. Uncertainty in heterogeneity estimates in meta-analyses. BMJ 2007;335:914-16.

22. Andrade SE, Raebel MA, Brown J, et al. Use of antidepressant medications during pregnancy: a multisite study. Am J Obstet Gynecol 2008;198:194-5.

23. Maenner MJ, Schieve LA, Rice CE, et al. Frequency and pattern of documented diagnostic features and the age of autism identification. J Am Acad Child Adolesc Psychiatry 2013;52:401-13. 